\title{
Cascaded Fiber-based Mid-Infrared Supercontinuum Source
}

This paper was downloaded from TechRxiv (https://www.techrxiv.org).

\section{LICENSE}

CC BY 4.0

SUBMISSION DATE / POSTED DATE

13-03-2020 / 26-03-2020

\section{CITATION}

Venck, Sébastien; St-Hilaire, François; Brilland, Laurent; Ghosh, Amar N.; Chahal, Radwan; Caillaud, Céline; et al. (2020): Cascaded Fiber-based Mid-Infrared Supercontinuum Source. TechRxiv. Preprint. https://doi.org/10.36227/techrxiv.11982267.v2

$\mathrm{DOI}$ 


\title{
Cascaded Fiber-based Mid-Infrared Supercontinuum Source
}

\author{
Sébastien Venck ${ }^{1}$, François St-Hilaire ${ }^{2,6}$, Laurent Brilland ${ }^{1}$, Amar N. Ghosh ${ }^{2}$, Radwan Chahal ${ }^{1}$, \\ Céline Caillaud ${ }^{1}$, Marcello Meneghetti ${ }^{3}$, Johann Troles ${ }^{3}$, Franck Joulain ${ }^{4}$, Solenn Cozic ${ }^{4}$, Samuel \\ Poulain $^{4}$, Guillaume Huss ${ }^{5}$, Martin Rochette ${ }^{6}$, John Dudley ${ }^{2}$, and Thibaut Sylvestre*2 \\ ${ }^{1}$ SelenOptics, Campus de Beaulieu, Rennes, France \\ ${ }^{2}$ Institut FEMTO-ST, CNRS UMR 6174, Université Bourgogne Franche-Comté, Besançon, \\ France \\ ${ }^{3}$ Université de Rennes, CNRS, ISCR-UMR 6226, Rennes, France \\ ${ }^{4}$ Le Verre Fluoré, Campus Kerlann, Bruz, France \\ ${ }^{5}$ LEUKOS, 37 rue Henri Giffard, Limoges, France \\ ${ }^{6}$ Department of Electrical and Computer Engineering, McGill University, Montréal, Québec, \\ Canada
}

March 13, 2020

\begin{abstract}
Mid-infrared supercontinuum (SC) sources in the 2 to $20 \mu \mathrm{m}$ molecular fingerprint region are in high demand for a wide range of applications including optical coherence tomography, remote sensing, molecular spectroscopy, and hyperspectral imaging. In this work, we investigate mid-IR SC generation in a cascaded silica-ZBLAN-chalcogenide fiber system directly pumped with a commercially-available 460-ps pulsed fiber laser operating in the telecommunications window at $1.55 \mu \mathrm{m}$. This fiber-based system is shown to generate a flat broadband mid-IR SC covering the entire range from 2 to $10 \mu \mathrm{m}$ with several tens of $\mathrm{mW}$ of output power. This technique paves the way for low cost, practical, and robust broadband SC sources in the mid-IR without the requirement of mid-infrared pump sources or Thulium-doped fiber amplifiers. We also describe a fully-realistic numerical model used to simulate the nonlinear pulse propagation through the cascaded fiber system and use our numerical results to discuss the physical processes underlying the spectral broadening in the cascaded system. We conclude with recommendations to optimize the current cascaded systems based on the simulation results.
\end{abstract}

*corresponding author: thibaut.sylvestre@univ-fcomte.fr 


\section{Introduction}

Fiber-based supercontinuum (SC) sources have become enormously useful in the last decade for a wide range of industrial and scientific applications $[1,2]$. New uses are also constantly emerging due to their unique properties that combine high brightness, multi-octave frequency bandwidth, fiber delivery and single-mode output. Applications include optical coherence tomography (OCT), material processing, chemical sensing, gas monitoring, broadband imaging, and absorption spectroscopy. Currently, there is a significant research effort focused on extending the wavelength coverage towards the mid-Infrared (Mid-IR) in the 2 to $20 \mu \mathrm{m}$ molecular fingerprint region [3-18]. Various soft glasses based on chalcogenide $\left(\mathrm{As}_{2} \mathrm{~S}_{3}, \mathrm{As}_{2} \mathrm{Se}_{3}, \mathrm{GeAsSe}\right)$ [3, 8], tellurite $\left(\mathrm{TeO}_{2}\right)$ [19], telluride (GeTe, GeAsTeSe) [20, 21], heavy-metal oxide $\left(\mathrm{PbO}-\mathrm{Bi}_{2} \mathrm{O}_{3}-\mathrm{Ga}_{2} \mathrm{O}_{3}-\mathrm{SiO}_{2}-\mathrm{CdO}\right)$ [10] and ZBLAN $\left(\mathrm{ZrF}_{4}-\mathrm{BaF}_{2}-\mathrm{LaF}_{3}-\mathrm{AlF}_{3}-\mathrm{NaF}\right)$ [23-26], have been used for drawing highly nonlinear infrared fibers, and experiments have shown efficient mid-IR SC generation up to $14 \mu \mathrm{m}$ in chalcogenide optical fibers [3] and up to $16 \mu \mathrm{m}$ in telluride fibers [21]. However, most of these mid-IR SC sources have been demonstrated using bulky mid-IR pump sources such as optical parametric oscillators (OPO) and amplifiers (OPA). Mid-IR fiber lasers and cascaded fiber systems have recently emerged as attractive and promising solutions for practical and commercial applications [25-35]. These fiber lasers-based systems indeed open routes to practical, table-top and robust mid-IR supercontinuum sources with high spectral power density. Of particular interest are cascaded fiber systems as they are pumped by standard pulsed fiber lasers at telecommunication wavelength. In cascaded SC generation, an initial pulsed fiber laser at a wavelength of $1550 \mathrm{~nm}$ is progressively red-shifted in a cascade of silica and soft-glass fibers, enabling a stepwise extension towards the Mid-IR. From a fundamental point of view, the overall objective is to strongly enhance the soliton self-frequency shift (SSFS) using dispersion-tailored highly nonlinear fiber segments to push forward the SC generation far in the mid-IR [29-31].

Among the most advanced fiber-based systems, Martinez et al. recently demonstrated a mid-IR SC from 2 to $11 \mu \mathrm{m}$ with $139 \mathrm{~mW}$ average power by concatenating solid-core ZBLAN, arsenic sulfide, and arsenic selenide fibers, pumped by a master oscillator power amplifier and three thulium-doped fiber amplifier stages [29,30]. Hudson et al. combined a $2.9 \mu \mathrm{m}$ ultrafast fiber laser based on holmium with an environmentally stable, polymer-protected chalcogenide fiber taper. By launching femtosecond, $4.2 \mathrm{~kW}$ peak power pulses into the $\mathrm{As}_{2} \mathrm{Se}_{3} / \mathrm{As}_{2} \mathrm{~S}_{3}$ tapered fiber, they demonstrated a SC spectrum spanning from 1.8 to $9.5 \mu \mathrm{m}$ with an average power of more than $30 \mathrm{~mW}$ [27]. C.R. Petersen et al. also demonstrated in 2016 Mid-IR SC generation beyond $7 \mu \mathrm{m}$ using a silica-fluoride-chalcogenide fiber cascade pumped by a $1.55 \mu \mathrm{m}$ seed laser and a thulium-doped fiber amplifier. By pumping a commercial $\mathrm{Ge}_{10} \mathrm{As}_{22} \mathrm{Se}_{68}$-glass photonic crystal fiber with $135 \mathrm{~mW}$ of the pump continuum from 3.5- $4.4 \mu \mathrm{m}$, they obtained a continuum up to $7.2 \mu \mathrm{m}$ with a total output power of $54.5 \mathrm{~mW}$, and $3.7 \mathrm{~mW}$ above $4.5 \mu \mathrm{m}$ [33]. Commercially available mid-IR SC source with an extended bandwidth up to $10 \mu \mathrm{m}$ and with $50 \mathrm{~mW}$ output power is now on sale at Norblis [36].

In this paper, we demonstrate mid-IR SC generation from to 2 to $10 \mu \mathrm{m}$ in a cascaded silica-ZBLAN$\mathrm{As}_{2} \mathrm{Se}_{3}$ fiber system directly pumped with a commercially-available 500-ps pulsed fiber laser at $1.55 \mu \mathrm{m}$ without any fiber amplifier stage. We provide details of the experimental cascaded fiber system and discuss the choice of the parameters such as chromatic dispersion and nonlinear coefficients used for optimal spectral broadening. We further report a fully-realistic numerical model based on cascaded generalized nonlinear Schrödinger equations with an adaptive step size method. The model includes all the linear and nonlinear responses of the fibers, the infrared filtering function, and the effective mode area variation across the full SC spectrum. We then use our numerical results to discuss the physical processes underlying the spectral broadening in the cascaded system, such as modulation instability (MI), soliton fission, dispersive wave generation, and intrapulse Raman scattering (IRS) [37,38]. We conclude with recommendations to optimize the current cascaded systems based on the simulation results. 


\section{$2 \quad$ Experimental Setup and Results}

A diagram of the experimental setup for cascaded mid-IR SC generation is shown in Figure 1(a). It consists of a concatenation of three commercially-available optical fibers including a $20 \mathrm{~cm}$-long singlemode silica fiber (SMF-28), a 25 m-long ZBLAN fiber, and a 9 m-long chalcogenide-glass photonic crystal fiber. Detailed specifications for each fiber segment are given in the next section including all parameters used in numerical simulations. This cascaded fiber arrangement was directly pumped by a compact (90 x $70 \times 15 \mathrm{~mm}^{3}$ only) pulsed fiber laser providing 460 ps-width pulse train at $100 \mathrm{kHz}$ repetition rate and at a center wavelength of $1550.6 \mathrm{~nm}$, a high peak power per pulse $(18 \mathrm{~kW})$, an average output power of $750 \mathrm{~mW}$, and a near diffraction limited randomly polarized beam $\left(\mathrm{M}^{2}<1.1\right)$. These compact lasers are mainly commercialized for range-finding and LIDAR applications. Light coupling between each fiber was achieved using high-numerical aperture aspheric lenses matching the fiber numerical apertures and their effective mode areas. A key element in the cascaded fiber system is a mid-IR long pass filter (LPF) placed in between the ZBLAN and the chalcogenide fibers. This filter rejects wavelengths below $1.9 \mu \mathrm{m}$. This prevents from two-photon absorption (TPA) and optical damage in the chalcogenide fiber, enabling a better long-term stability of the mid-IR SC source. The long-pass filter is a Germanium (Ge) Arcoated broadband window inserted in between two aspheric lenses. The generated SC spectra have been recorded using a mid-IR optical spectrometer including a monochromator (ORIEL 7240) and a highly sensitive $\mathrm{Hg}-\mathrm{Cd}-\mathrm{Te}$ detector. The chalcogenide fiber output face was terminated with an end cap fiber and connectorized using an $\mathrm{FC} / \mathrm{PC}$ connector for practical applications.

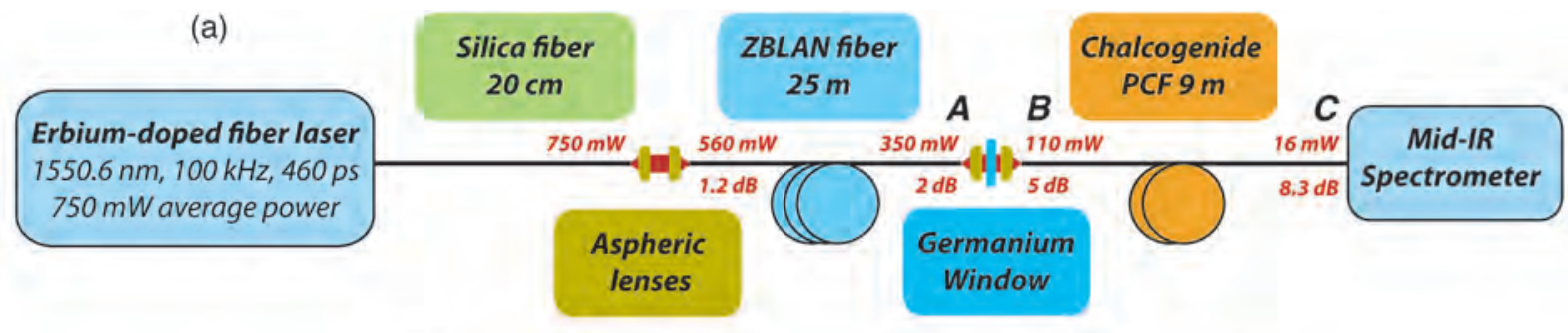

(b)

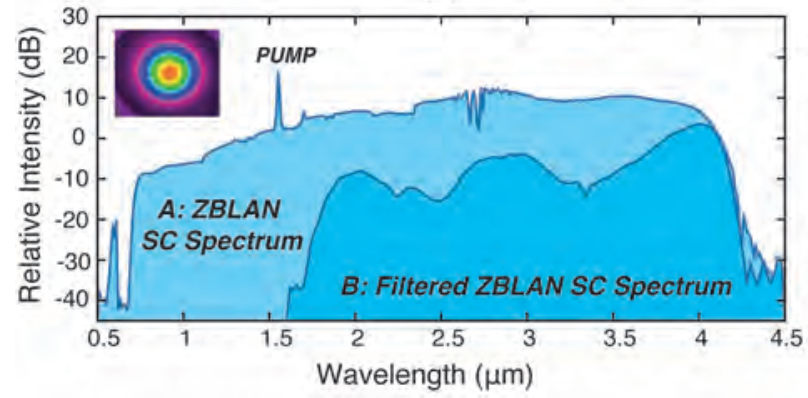

(c)

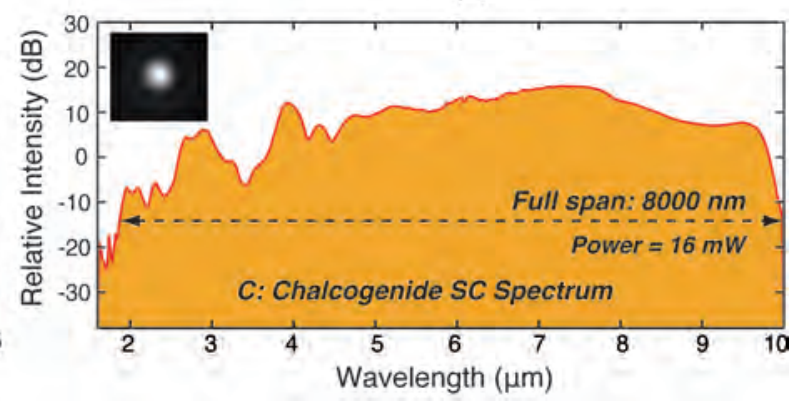

Figure 1: (a) Experimental setup for mid-infrared SC generation in a cascaded silica-ZBLAN-chalcogenide optical fiber system. (b) Experimental SC spectra at the ZBLAN fiber output (light blue) and after the long-pass filter (dark blue). (c) Experimental SC spectrum at the chalcogenide fiber output (yellow). The insets show the optical mode profiles out of both the ZBLAN (c) and chalcogenide (c) optical fibers.

We provide experimental data for the spectra measured at three key points in the cascaded fiber setup $(\mathrm{A}, \mathrm{B}, \mathrm{C})$. The first spectrum, shown in Figure 1(b) (light blue), was measured at the output of the ZBLAN fiber at point A, before the long-pass infrared filter. The supercontinuum spans from $0.7 \mu \mathrm{m}$ up to $4.1 \mu \mathrm{m}$. The inset in Fig. 1(b) shows the ZBLAN fiber's mode profile measured with an IR camera. The second 
spectrum, also shown in figure 1(b) (dark blue), was measured after the filter at point B. This is the filtered IR spectrum injected into the chalcogenide fiber. The final SC spectrum at the output of the chalcogenide fiber is shown in figure 1(c) in yellow. As can be seen, the full SC spans from $2 \mu \mathrm{m}$ up to $9.8 \mu \mathrm{m}$ with a relatively smooth and flat bandwidth. The measured spectrum however shows a decrease in spectral intensity for shorter wavelengths and significant modulations in intensity (especially from 2 to $4 \mu \mathrm{m}$ ). Our simulations will show that those are not entirely a result of SC dynamics, but presumably artefacts from the wavelength sensitivity of the spectrometers used for measurement. The measured average power at the output is $16 \mathrm{~mW}$, which corresponds to roughly $2 \%$ of the pump's average power $(750 \mathrm{~mW})$. Significant losses occur in the cascaded system, mainly from the free-space optics between the ZBLAN fiber and the chalcogenide fiber, including Fresnel reflections and coupling losses due to mode field diameter mismatch, aspheric lenses, cleaving imperfections, and optical misalignment. All these losses and the output power of every stage of the cascade fiber system are mentioned in red in figure 1(a). Despite this low conversion efficiency, the brightness of this source of a few microwatt per nanometer is sufficient for practical applications. It is worth mentioning that the experimental spectra are provided only at maximum pump power $(750 \mathrm{~mW})$ at the output of every fiber-stage, which look very flat and therefore hard to tell what actual broadening mechanism is dominant. However, we have observed that when decreasing the input pump laser current (from $4600 \mathrm{~mA}$ to $3600 \mathrm{~mA}$ ), the $\mathrm{SC}$ in the ZBLAN fiber considerably reduces its lateral extension at long wavelength from $4.2 \mathrm{~m}$ to $3.5 \mathrm{~m}$, thus limiting the additional extension in stages in the chalcogenide fiber. In such a cascade system, it is indeed crucial to obtain the most efficient $\mathrm{SC}$ extension in the ZBLAN fiber, as we will see thereafter using numerical simulations.

\section{$3 \quad$ Numerical Method}

To simulate nonlinear pulse propagation in the cascaded fiber system, we used the generalized nonlinear Schrödinger equation (GNLSE) and solved the propagation equation numerically with the split-step Fourier method (SSFM) [37,38] combined with an adaptive step size [39]. The GNLSE can be written in the following form:

$$
\frac{\partial A(z, T)}{\partial z}=-\frac{\alpha}{2} A+\mathrm{i} \sum_{k=2}^{\infty} \frac{\mathrm{i}^{k} \beta_{k}}{k !} \frac{\partial^{k}}{\partial T^{k}} A+\mathrm{i} \gamma\left(1+\mathrm{i} \tau_{0} \frac{\partial}{\partial T}\right)\left[A \cdot R(T) \circledast|A|^{2}\right]
$$

This equation models the evolution of the complex pulse envelope $A(z, T)$ as it propagates inside an optical fiber in a frame of reference moving with the group velocity of the pulse: $T=t-\beta_{1} z$. The first term on the right models optical losses with the linear loss coefficient $\alpha$. The second term models dispersion with a Taylor expansion of the propagation constant $\beta(\omega)$, where $\beta_{k}=\frac{\partial^{k} \beta(\omega)}{\partial \omega^{k}}$. The third term models Kerr nonlinearity, including the Raman response of the material and a first-order correction for the frequency

dependence of the nonlinear parameter $\gamma, \gamma(\omega)=\frac{\omega n_{2}(\omega)}{c A_{\text {eff }}(\omega)}$. The first-order correction is referred to as the shock term and is characterized by a time-scale $\tau_{0}$, which can include the frequency-dependence of $A_{\text {eff }}$, $n_{2}$, and $n_{\text {eff }}$ (see [40] for more details).

$$
\tau_{0}=\frac{1}{\omega_{0}}-\left[\frac{1}{n_{\mathrm{eff}}(\omega)} \frac{\mathrm{d} n_{\mathrm{eff}}}{\mathrm{d} \omega}\right]_{\omega_{0}}-\left[\frac{1}{A_{\mathrm{eff}}(\omega)} \frac{\mathrm{d} A_{\mathrm{eff}}}{\mathrm{d} \omega}\right]_{\omega_{0}}+\left[\frac{1}{n_{2}(\omega)} \frac{\mathrm{d} n_{2}}{\mathrm{~d} \omega}\right]_{\omega_{0}}
$$

The nonlinear response of the fiber is modeled with a convolution (denoted by $\circledast$ ) of the nonlinear response function $R(T)$ and the pulse power profile $|A(z, T)|^{2} . R(T)$ is commonly divided into an instantaneous electronic response (Kerr) and a delayed molecular response (Raman), and reads as $R(T)=\left(1-f_{R}\right) \delta(T)+$ $f_{R} h_{R}(T)$, where $f_{R}$ is the fractional contribution of the delayed Raman response, $\delta(T)$ is the Dirac delta function that models the instantaneous Kerr response, and $h_{R}(T)$ is the delayed Raman response function. 
$R(T)$ is normalized such that $\int_{-\infty}^{\infty} R(T) \mathrm{d} T=1$. The Raman response function $h_{R}(T)$ is modeled using two characteristic times related to phonon dynamics in the material, $\tau_{1}$ and $\tau_{2}$ (see Ref [38] for more details):

$$
h_{R}(T)=\left(\tau_{2}^{-2}+\tau_{2}^{-2}\right) \tau_{1} \exp \left(-T / \tau_{2}\right) \sin \left(T / \tau_{1}\right)
$$

We will use Eq. (3) for both the silica and chalcogenide fibers (See Table 1 for parameters). However, as the ZBLAN fiber has a dual-peak Raman gain spectrum, we will use another model based on Eq. (5). A crucial step in modeling nonlinear pulse propagation in a cascaded fiber system is defining the longitudinal step size $h$. To accurately model the effects of nonlinearity and dispersion, $h$ must be much smaller than both the dispersion length and the nonlinear length, defined as, $L_{\mathrm{NL}}=\frac{1}{\gamma P_{0}}$ and $L_{\mathrm{D}}=\frac{T_{0}^{2}}{\left|\beta_{2}\right|}$, respectively, where $P_{0}$ is the peak power, and $T_{0}$ is the $1 / e$ pulse width. These two conditions must hold throughout the propagation length. Pumping with a quasi-continuous-wave laser (i.e. 460 ps pulses) leads to the break-up of the initial pulse via modulation instability (MI) into a vaste collection of ultrashort soliton-like pulses with variable peak powers $P_{0}$ and durations $T_{0}$. The choice of $h$ is therefore not as straightforward in this case. Our simulations show that the nonlinear length $L_{\mathrm{NL}}$ is a much more limiting factor than the dispersion length $L_{\mathrm{D}}$ for the step size $h$ because of the high peak power reached within the soliton train. When the soliton train starts propagating, the maximal peak power varies significantly along the propagation distance $z$ because of randomly occurring soliton collisions. This means that the condition $h<<L_{\mathrm{NL}}$ can change drastically along $z$. To account for this, we used an adaptive step size method for the SSFM algorithms: the nonlinear phase-rotation method (see [39] for more details). This method consists of limiting the nonlinear phase-shift $\phi_{\mathrm{NL}}=\gamma P_{0} h$ to a sufficiently small value at each iteration, i.e. the step size $h$ is adapted at each iteration to optimize computing time and accuracy. For a train of soliton pulses, the maximal phase-shift is calculated from the maximal soliton peak power $P_{0}^{\max }$.

Every iteration $i$, the step size $h(i)$ is determined by $h(i)=\frac{\phi_{\mathrm{NL}}^{\max }}{\gamma_{0} P_{0}^{\max }(i)}$. For our simulations, we found that a maximal nonlinear phase-shift of $\phi_{\mathrm{NL}}^{\max }=1 / 50 \mathrm{rad}$ is enough for convergence and allows for reasonable computing times.

\subsection{Cascaded fiber system}

This section provides all linear and nonlinear parameters of the cascaded fiber system used in numerical simulations. The first fiber segment consists of a length of $20 \mathrm{~cm}$ of standard single-mode silica fiber (SMF-28). Although this fiber plays little role in SC broadening, it serves as a nonlinear modulation instability stage that triggers the soliton dynamics in both the ZBLAN and chalcogenide fibers. The nonlinear parameters for fused silica fiber are taken from [38] and are shown in Table 1.

Table 1: Nonlinear parameters for the cascaded fiber system

\begin{tabular}{|c|c|c|c|c|c|c|c|}
\hline Fiber & $\mathrm{L}(\mathrm{cm})$ & $n_{2}\left(\mathrm{~cm}^{2} / \mathrm{W}\right)$ & $\mathrm{A}_{\text {eff }}\left(\mu \mathrm{m}^{2}\right)$ & $\gamma\left(\mathrm{W}^{-1} \mathrm{~km}^{-1}\right)$ & $f_{R}$ & $\tau_{1}(\mathrm{fs})$ & $\tau_{2}(\mathrm{fs})$ \\
\hline SMF-28 & 20 & $2.4 \cdot 10^{-16}$ & 85 & 1.3 & 0.18 & 12.2 & 32 \\
\hline ZBLAN & 2500 & $2.1 \cdot 10^{-16}$ & 43 & 2 & 0.2 & Eq. $(5)$ & Eq. $(5)$ \\
\hline Chalcogenide & 900 & $1.1 \cdot 10^{-13}$ & 62 & 720 & 0.1 & 23.2 & 195 \\
\hline
\end{tabular}

The wavelength dependence of $\mathrm{A}_{\text {eff }}$ and $n_{2}$ is negligible over the spectral bandwidth along this first fiber segment. The dispersion characteristics of SMF-28 are modeled using a Taylor series expansion. The following values are used: $\beta_{2}=-21.4 \cdot 10^{-27} \mathrm{~s}^{2} / \mathrm{m}, \beta_{3}=0.12 \cdot 10^{-39} \mathrm{~s}^{3} / \mathrm{m}$ (from [38]). The $1550 \mathrm{~nm}$ pump is in the anomalous dispersion regime. Optical losses were neglected for this fiber segment given the short length $(20 \mathrm{~cm})$ and low absorption of silica fibers at $1550 \mathrm{~nm}(0.2 \mathrm{~dB} / \mathrm{km})$. 
The second fiber consists of a length of $25 \mathrm{~m}$ of a commercially available step-index fluoride (ZBLAN) fiber from Le Verre Fluoré [41]. The core and cladding diameters are $8.5 \mu \mathrm{m}$ and $125 \mu \mathrm{m}$, respectively. The refractive index of core and cladding are plotted in Figure 2(a) and the dispersion curve is shown in Fig. 2(b) in blue. Experimental data of dispersion from 1.2 to $2.2 \mu \mathrm{m}$ are provided in red dots. To obtain the dispersion of the fiber over the full bandwidth, we solved the characteristic equation for a step-index fiber with the wavelength-dependent core and cladding indices shown in figure 2(a). Results show that the zero dispersion wavelength (ZDW) is $1525 \mathrm{~nm}$. Therefore the $1550 \mathrm{~nm}$ pump wave is in the slightly anomalous dispersion regime.

(a)

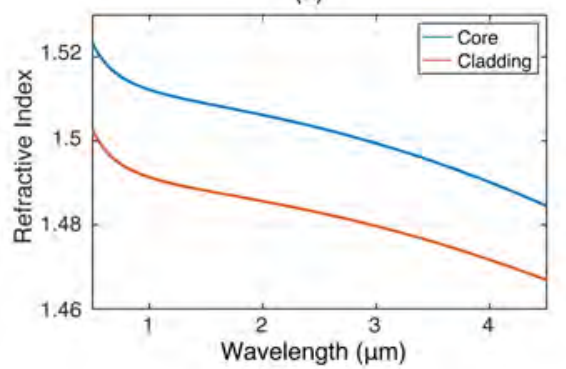

(c)

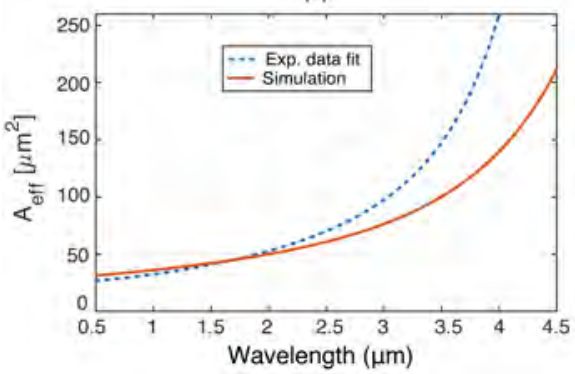

(b)

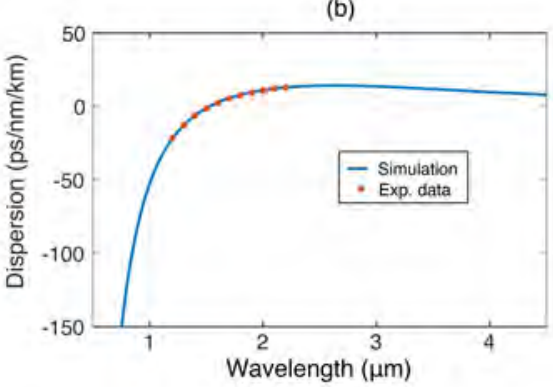

(d)

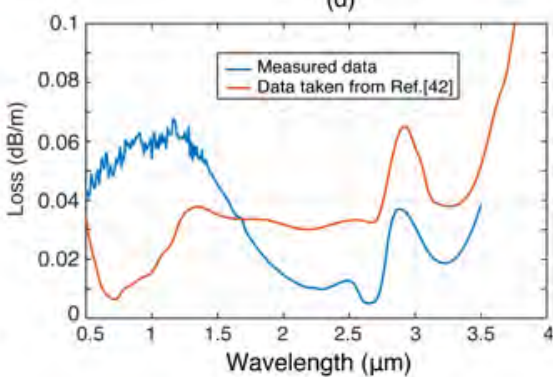

Figure 2: ZBLAN step-index optical fiber specifications: (a) Core (blue) and cladding (Red) refractive indices versus wavelength. (b) Computed dispersion (blue curve) and experimental data (Red dots). (c) Simulated (red) and experimental (blue) effective mode area. (d) Loss spectrum: blue: Measured data, red: data from [42].

The strong effective mode area variation from 0.5 to $5 \mu \mathrm{m}$ was also considered. We calculated $\mathrm{A}_{\text {eff }}$ over the same bandwidth by solving the characteristic equation for a step-index fiber with the wavelengthdependent core and cladding indices. The results are compared with the experimental data in Fig. 2(c). Our simulated results were used instead of the measured data because it provided a better fit with the experimental SC when the wavelength dependence of $\mathrm{A}_{\text {eff }}$ was included.

Figure 2(d) shows the optical losses of the ZBLAN fiber. The measurements are shown in blue, and the data used in the simulations (taken from [42]) is shown in orange. The data from [42] was used in our simulations to better model the limit of the transmission window in the infrared, which ends between 4 and $4.5 \mu \mathrm{m}$. As we will show in our results, the transmission window limit of the ZBLAN fiber is the only feature of the loss curve that has a significant impact on spectral nonlinear dynamics. The small discrepancies with the measured loss curve should not affect the overall SC mechanisms.

The nonlinear parameters for the ZBLAN fiber are listed in Table 1. The wavelength dependence of the nonlinear index is neglected. The Raman response function $h_{R}$ was obtained with the model developed in Ref. [24], where the measured Raman gain spectrum $g_{R}(\Omega)$ is approximated by a sum of two Gaussian 
functions, and the response function is extracted from the imaginary part of the Fourier transform as

$$
h_{R}(T>0)=C \cdot \int_{0}^{\infty} g_{R}(\Omega) \sin (\Omega T) \mathrm{d} \Omega
$$

where $C$ is a normalization constant chosen such that $\int_{-\infty}^{\infty} h_{R}(T) \mathrm{d} T=1$. The following equation was used in our simulations to model $g_{R}(\Omega)$ :

$$
g_{R}(\Omega)=a_{1} \exp \left(\frac{\left(\Omega /(2 \pi)-\nu_{1}\right)^{2}}{2 \omega_{1}^{2}}\right)+a_{2} \exp \left(\frac{\left(\Omega /(2 \pi)-\nu_{2}\right)^{2}}{2 \omega_{2}^{2}}\right)
$$

with $a_{1}=0.54 \cdot 10^{-11} \mathrm{~cm} / \mathrm{W}, a_{2}=0.25 \cdot 10^{-11} \mathrm{~cm} / \mathrm{W}, \nu_{1}=17.4 \mathrm{THz}, \nu_{2}=12.4 \mathrm{THz}, \omega_{1}=0.68 \mathrm{THz}$, $\omega_{2}=3.5 \mathrm{THz}[24]$.

The third fiber sample is a chalcogenide-glass solid-core microstructured optical fiber. It was fabricated from $\mathrm{As}_{38} \mathrm{Se}_{62}$ glass using the casting method [45]. It has a core diameter of $11.2 \mu \mathrm{m}$, a pitch of $7.11 \mu \mathrm{m}$, and an air-hole diameter of $3.23 \mu \mathrm{m}$. Figure 3 shows a cross section of the microstructure with dimensions for the air hole structure (a) as well as the refractive index of $\mathrm{As}_{38} \mathrm{Se}_{62}$ glass (b). The following Sellmeier model was used, $n^{2}=A_{0}+\frac{A_{1} \lambda^{2}}{\lambda^{2}-a_{1}^{2}}++\frac{A_{2} \lambda^{2}}{\lambda^{2}-a_{2}^{2}}$, with $A_{0}=3.7464, A_{1}=3.9057, A_{2}=0.9466, a_{1}=0.4073 \mu \mathrm{m}$, and $a_{2}=40.082 \mu \mathrm{m}$. These values were chosen to obtain the best fit with the refractive index measured data shown in Fig.3(a).
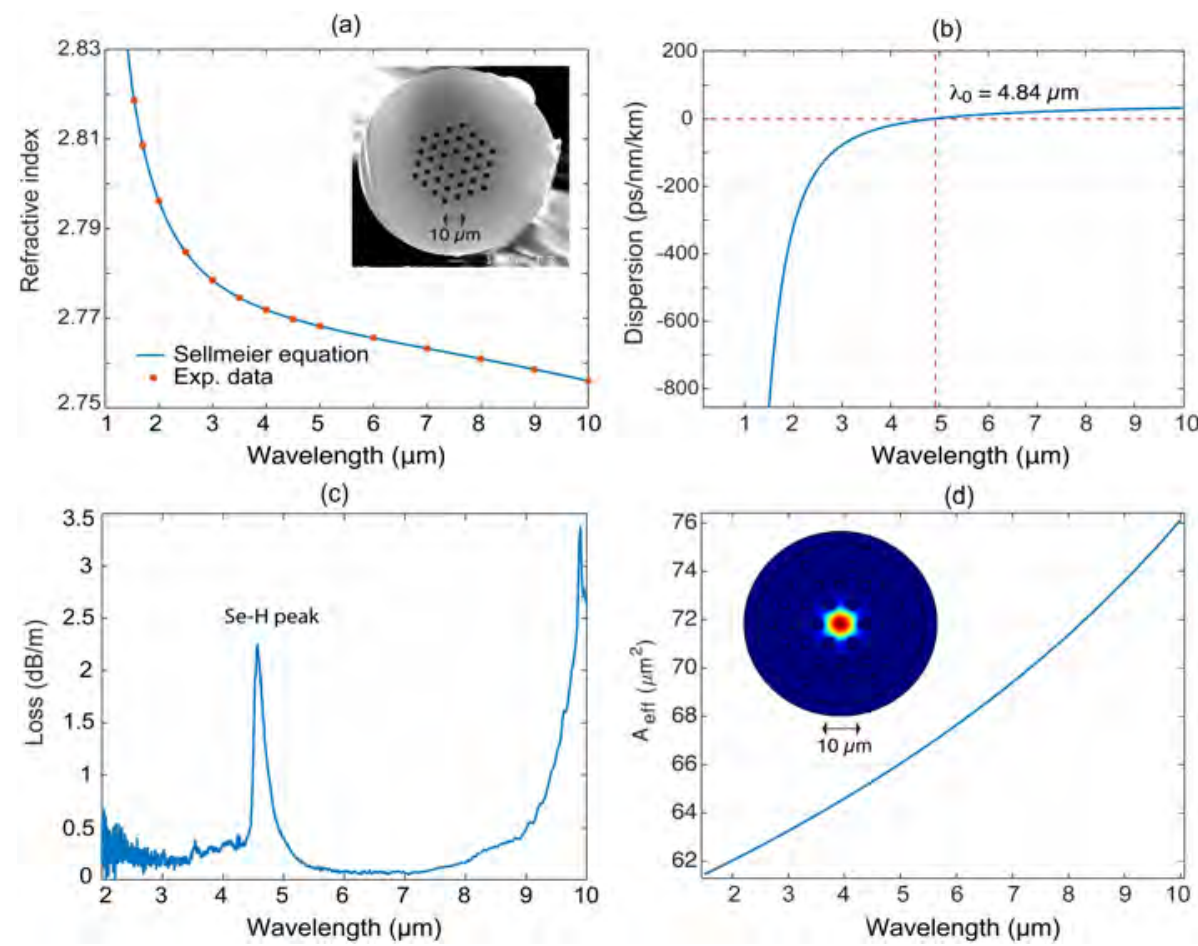

Figure 3: (a) Refractive index of $\mathrm{As}_{38} \mathrm{Se}_{62}$ glass calculated with the Sellmeier model. Inset shows the cross-section image of the microstructured chalcogenide fiber. (b) Computed dispersion curve for the microstructured chalcogenide fiber with a zero dispersion wavelength near $5 \mu \mathrm{m}$. (c) Absorption loss of the chalcogenide-based microstructured fiber. (d) Computed effective mode area. Inset shows the fundamental optical $\mathrm{HE}_{11}$ mode. 
The dispersion curve for the fundamental mode was calculated by the finite-element method using the COMSOL software. The result is shown in Fig. 3(a). The zero-dispersion wavelength is $4.84 \mu \mathrm{m}$. The calculated effective mode area is shown in figure 3(d). Optical losses for the chalcogenide fiber are shown in figure 3(c). The transmission window ends near $10 \mu \mathrm{m}$, and there is an absorption peak around $4.56 \mu \mathrm{m}$ due to the presence of Se-H chemical bounds in the glass. The nonlinear parameters considered for this $\mathrm{As}_{38} \mathrm{Se}_{62}$ glass fiber are summarized in table 1. The nonlinear index $n_{2}$ is very large and is taken from $[45,46]$. The Raman response is modelled with equation (3) and the Raman parameters are taken from [27]. This gives Raman gain peak centered around $6.9 \mathrm{THz}$ with a linewidth of about $3.2 \mathrm{THz}$.

\subsection{Infrared Optical Filter}

The short-wavelength filtering of the ZBLAN fiber output is achieved experimentally with a combination of a germanium window and lenses with IR anti-reflective (AR) coatings for infrared. This filtering window aims to cut all the energy $<1.9 \mu \mathrm{m}$ in the ZBLAN fiber output spectrum to prevent two-photon absorption and optical damage in the chalcogenide optical fiber. The global transmission function of this filtering system was modelled with a supergaussian function of the following form,

$$
T=\exp \left(-\left(\frac{\lambda-\Lambda_{0}}{\Delta \lambda}\right)^{m}\right)
$$

The parameters $\Lambda_{0}, m$, and $\Delta \lambda$ where adjusted to obtain the best fit with the experimental spectrum measured after the filtering system. The following values where used: $\Lambda_{0}=11.87 \mu \mathrm{m}, \mathrm{m}=80$, and $\Delta \lambda=10 \mu \mathrm{m}$.

\section{Simulation Results}

In this section, we present simulation results for three different pump pulse durations: $50 \mathrm{ps}, 200 \mathrm{ps}$, and $460 \mathrm{ps}$ (the measured experimental duration). All the spectral and temporal evolutions shown thereafter are plotted with colormaps of relative intensity with a $80 \mathrm{~dB}$ range from blue to red (See figure 4). For the simulations, a Gaussian pulse was used. Quantum noise was added to the initial pulse following the one photon per mode model (see [44] for more details). The numerical models used for each fiber are also presented, including dispersion, nonlinear parameters and losses. A peak power of $6 \mathrm{~kW}$ was chosen for the three pump pulses to get the best match with experimental results. For this type of simulation, we typically use a lower peak power than the experimental peak power, which is around $18 \mathrm{~kW}$ in this case. This is due to the fact that the simulation model considers perfectly polarized and single-mode propagation. In experimental conditions, random polarization and multi-mode behavior is generally detrimental to spectral broadening mechanisms, hence the need for more peak power than in simulations. Simulations were carried out for the three pump pulse durations in the SMF-28 fiber and the ZBLAN fiber. As expected from the theory of SC generation with quasi-CW pulses, we show that the same mechanisms occur for the three different pump pulse durations, and that the experimental SC bandwidths at the output of the ZBLAN and chalcogenide fibers can be achieved with pump pulses as short as 50 ps. The SSFM algorithm requires high longitudinal precision in the chalcogenide fiber because of high nonlinearity $\left(\gamma_{0}=720 \mathrm{~km}^{-1} \mathrm{~W}^{-1}\right)$. As a result, simulations for $200 \mathrm{ps}$ and $460 \mathrm{ps}$ pump pulses, which require at least $\mathrm{Nt}=2^{21}$ sample points, would require unrealistic computing times. Therefore, simulations in the chalcogenide fiber were only carried out with the intermediate ZBLAN results from the 50 ps pump pulse. 


\subsection{Silica fiber}

The simulation results for $20 \mathrm{~cm}$ of propagation in the single-mode silica fiber (SMF-28) are shown in figure 4 in colormap and for a $460 \mathrm{ps}$ pulse. The pulse shape remains virtually unchanged throughout this fiber segment. The only notable feature is the apparition of weak MI Stokes and anti-Stokes sidebands in the spectrum around 1.51 and $1.59 \mu \mathrm{m}$, respectively. They can be seen appearing on either side of the pump in the spectral evolution, as shown in top of figure 4(b). Their specific frequencies relative to the pump $\left(\Omega_{\mathrm{MI}}=\omega_{P}-\omega_{S}\right)$ are given by the scalar phase-matching relation that reads as $\beta_{2} \Omega_{\mathrm{MI}}^{2}+\frac{\beta_{4}}{12} \Omega_{\mathrm{MI}}^{4}+2 \gamma P_{0}=0$, Where $P_{0}$ is the peak power, $\gamma$ is the nonlinear parameter, $\beta_{2}$ is the GVD parameter, and $\beta_{4}$ is the fourthorder dispersion [38]. A more detailed view is shown in Figure 6. It is worth noting, however, that our simulations show this first fiber segment has little to no effect on the overall SC evolution in the ZBLAN and chalcogenide fibers.

(a)

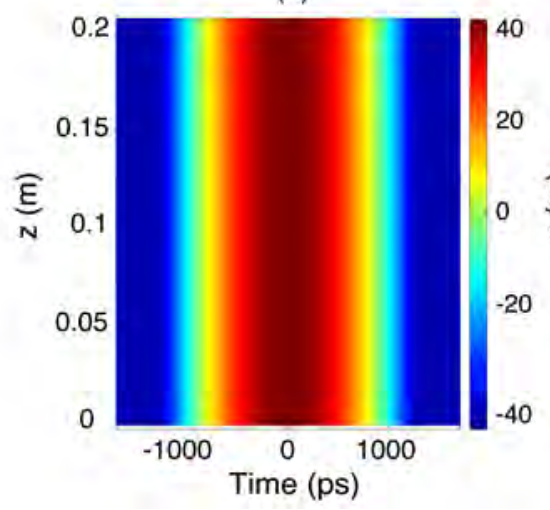

(b)

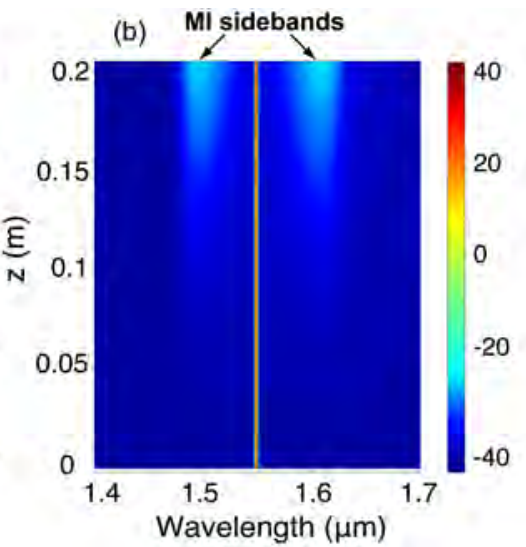

Figure 4: (a) Temporal and (b) spectral evolution in the silica fiber (SMF-28) for a 460 ps pulse and a 6 $\mathrm{kW}$ peak power.

\subsection{ZBLAN Fiber}

The simulated spectral and temporal evolutions in both the silica and the ZBLAN fibers are shown in figure 5 . In the bottom, the final spectrum after $25 \mathrm{~m}$ of propagation is compared to the experimental spectrum. The agreement is particularly excellent. This is due to the fact that the total bandwidth and soliton selffrequency shift (SSFS) are only limited by the transmission window of the fiber in the mid-infrared which ends between 4 and $4.5 \mu \mathrm{m}$ (see figure $2(\mathrm{~d})$ ). The break-up of the pulse into a train of solitons can be seen from $2 \mathrm{~m}$ in Fig. 5(b). Beyond this point, the continuous Raman-induced self-frequency shift of the solitons is clearly seen in both the spectral and temporal evolutions as they gradually separate from the main pulse due to their dispersion increasing with the SSFS. Events of soliton collision can also be observed in the spectral evolution. They manifest themselves as sharp increases in the spectrum bandwidth on the long-wavelength side (e.g. around $16 \mathrm{~m}$ in the $50 \mathrm{ps}$ spectral evolution). Dispersive waves can be seen rapidly drifting away from the main pulse in the time evolution beyond $1000 \mathrm{ps.} \mathrm{In} \mathrm{terms} \mathrm{of} \mathrm{bandwidth,}$ the simulation provide a nearly perfect match with the experimental spectrum (within $0.05 \mu \mathrm{m}$ at the $-20 \mathrm{~dB}$ level of the maximum intensity). 

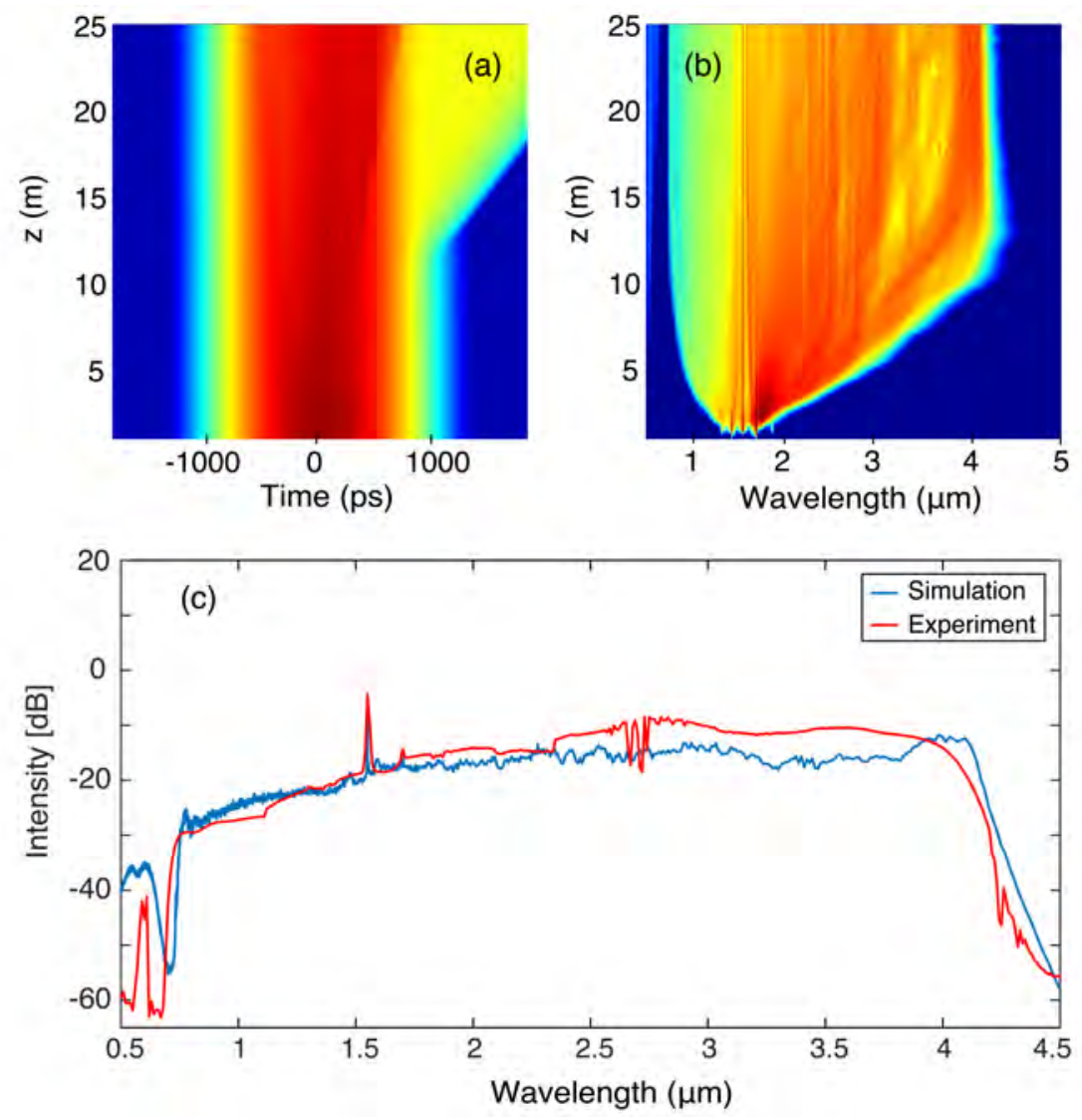

Figure 5: (a) Spectral and (b) time-domain evolution for the 460 ps pump pulse with the output experimental and numerical spectra shown in (c).

From Fig.5(b), we see that the SC in ZBLAN reached a maximum extension at $4.2 \mu \mathrm{m}$ around $13 \mathrm{~m}$. At longer distance, the SC becomes slightly narrower due to the absorption edge around 4-4.2 $\mu \mathrm{m}$ in ZBLAN, and since there is not much spectral evolution between $15 \mathrm{~m}$ and $25 \mathrm{~m}$ in the ZBLAN fiber, this might indicate that the peak power of the solitons are not very high, nonlinear effects are not very important and the dispersion continue to increase the pulse duration of these solitons. Therefore, it might be more efficient to cut the ZBLAN fiber at $13 \mathrm{~m}$ to $15 \mathrm{~m}$ to inject its output SC (which is broader and maybe more intense soliton train) in the following chalcogenide fiber.

To gain insight on the processes initiating SC generation in the ZBLAN fiber, we show in figure 6 the details of the first $50 \mathrm{~cm}$ of spectral evolution. The strongest sidebands correspond to the MI-enabled Stokes and anti-Stokes Raman sidebands around 1.42 and $1.70 \mu \mathrm{m}$. Around 1.51 and $1.59 \mu \mathrm{m}$ are the residual MI sidebands from the silica fiber. Weak MI ZBLAN sidebands are visible around 1.39 and $1.755 \mu \mathrm{m}$. Each of the wavelength pairs given here and identified on the plot were calculated theoretically. The MI wavelengths can be derived from the same equation as for the silica fiber, and the Raman wavelengths correspond to the peak of the ZBLAN Raman gain at $17.4 \mathrm{THz}$. 


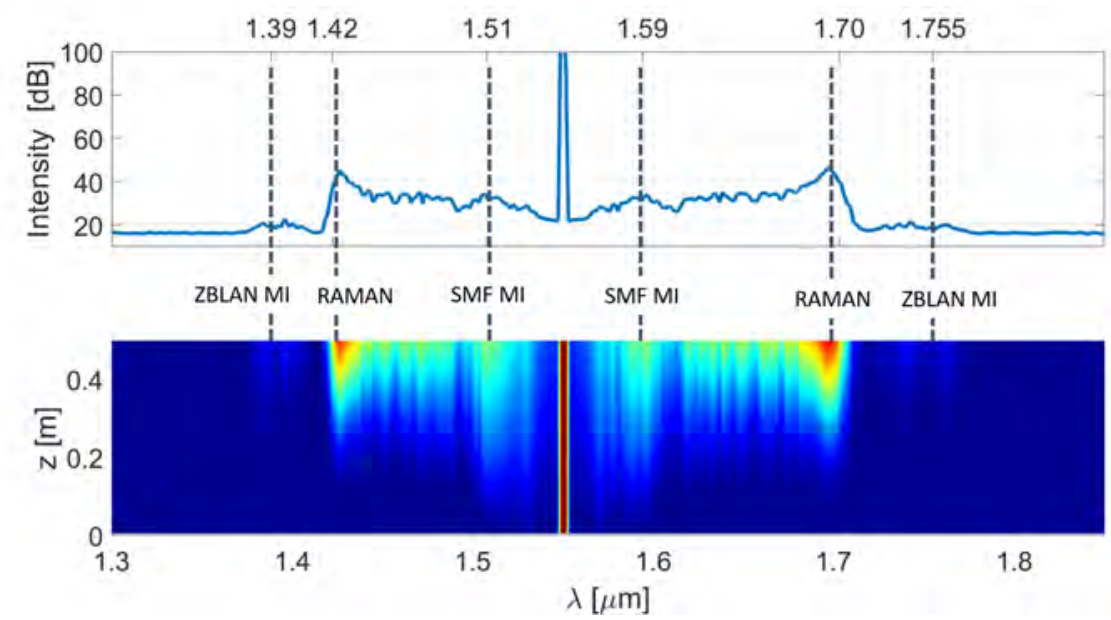

Figure 6: Initial spectral evolution in the ZBLAN fiber. The wavelengths marked with the dotted lines correspond to the theoretical MI and Raman wavelengths calculated with phase-matching equation in the text and the Raman gain peak at $17.4 \mathrm{THz}$, respectively.

From theory and simulation, we confirm that the time and spectral domain evolutions of the $460 \mathrm{ps}$ pulse is well approximated by the 50 ps pulse. Longer pulses simply provide a longer collection of solitons but that will not change the statistical distribution of peak powers and pulse widths. Therefore, from now on, simulations are carried out only with the $50 \mathrm{ps}$ pump pulse to save computing time. Figure 7 shows the filtered simulated spectrum compared with the experimental filtered spectrum using 50-ps input pulse. This is the spectrum injected in the chalcogenide fiber.

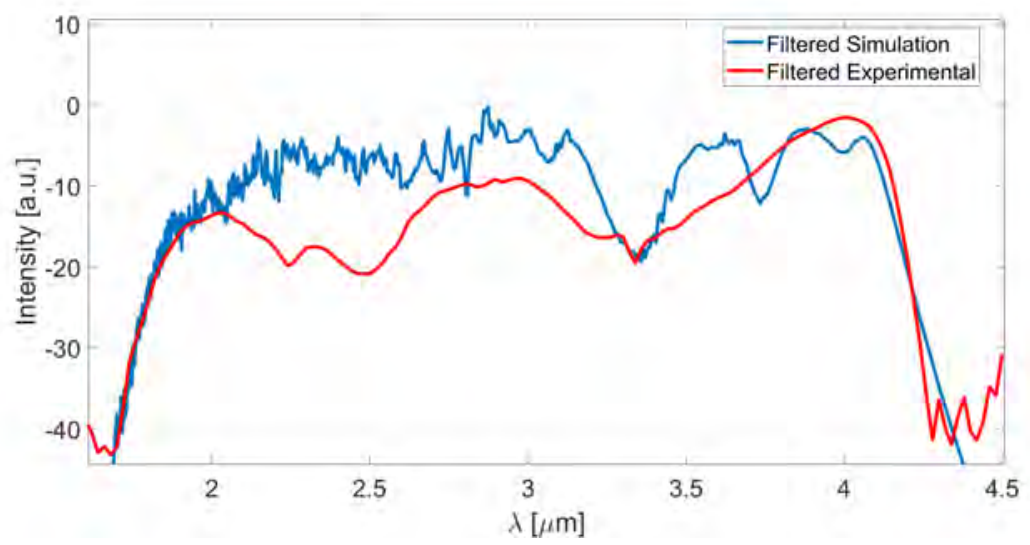

Figure 7: Filtered spectrum injected in the chalcogenide fiber.

\subsection{Chalcogenide Fiber}

The simulation results for the first $2 \mathrm{~cm}$ of propagation in the chalcogenide fiber are shown in Fig. 8. The injected spectrum from the filtered ZBLAN output lies entirely in the normal dispersion regime of the chalcogenide fiber which has its ZDW at $4.838 \mu \mathrm{m}$ (marked by the dotted line). The initial spectral evolution shows that, from the train of thousands of pulses injected in the chalcogenide fiber, a fraction of them have a small enough width $T_{0}$ to drift through the zero-dispersion via intrapulse Raman scattering 
(IRS). The pulses crossing the ZDW evolve into solitons and create an initial low-intensity continuum on the long-wavelength side through SSFS.

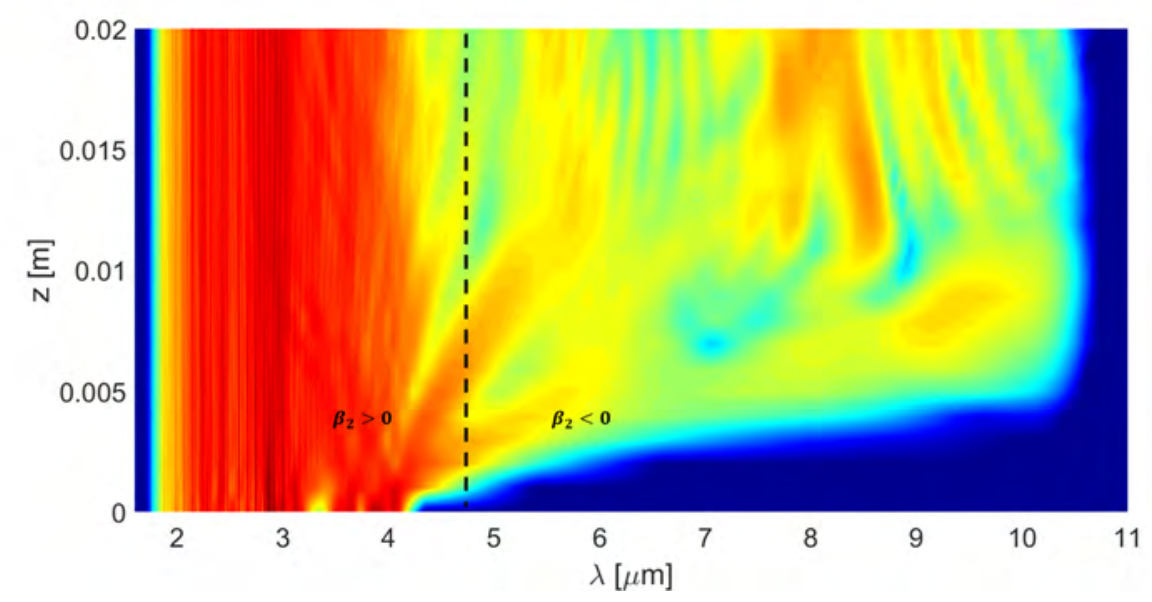

Figure 8: Initial spectral evolution in the chalcogenide fiber.

The spectral evolution over the full length of chalcogenide fiber is shown in figure 9 . The top of the figure shows the final output spectrum compared to the experimental spectrum. In terms of spectral bandwidth, our numerical result matches nearly perfectly the experimental measurement. If we compare the numerical and experimental SC bandwidth at $-20 \mathrm{~dB}$ of maximum intensity, we get a deviation of only $0.04 \mu \mathrm{m}$ over the full $8 \mu \mathrm{m}$ width. As for the ZBLAN fiber, spectral broadening in the chalcogenide fiber is only limited by its transmission window and the confinement losses.

The spectral evolution of figure 9 shows interesting SC dynamics. From 0 to $5 \mathrm{~m}$, most of the energy remains in the normal dispersion regime $\left(\beta_{2}>0\right)$. Energy rapidly accumulates at the limit of the transmission window (around $9.5 \mu \mathrm{m}$ ) from the pulses that initially crossed the ZDW. These solitons tend to accumulate around this wavelength as their SSFS is stopped by the upper limit of the transmission window. The effect of the absorption peak at around $4.6 \mu \mathrm{m}$ (see figure 3) can be clearly seen on the lowenergy continuum. From the beginning, the main train of pulses can be seen drifting through intrapulse Raman scattering towards the ZDW. After $5 \mathrm{~m}$ most of the pulses remain trapped by the ZDW barrier and stop their frequency drift. From $5 \mathrm{~m}$ onwards, a fraction of the pulses cross the ZDW barrier and start evolving as solitons on the anomalous dispersion side $\left(\beta_{2}<0\right)$. This gradually adds energy to the initial low-energy continuum via the SSFS of the solitons. The spectrum evolves towards thermalization, where energy is equally distributed throughout the transmission bandwidth. The output is therefore a relatively flat spectrum from 2 to $9.7 \mu \mathrm{m}$. 


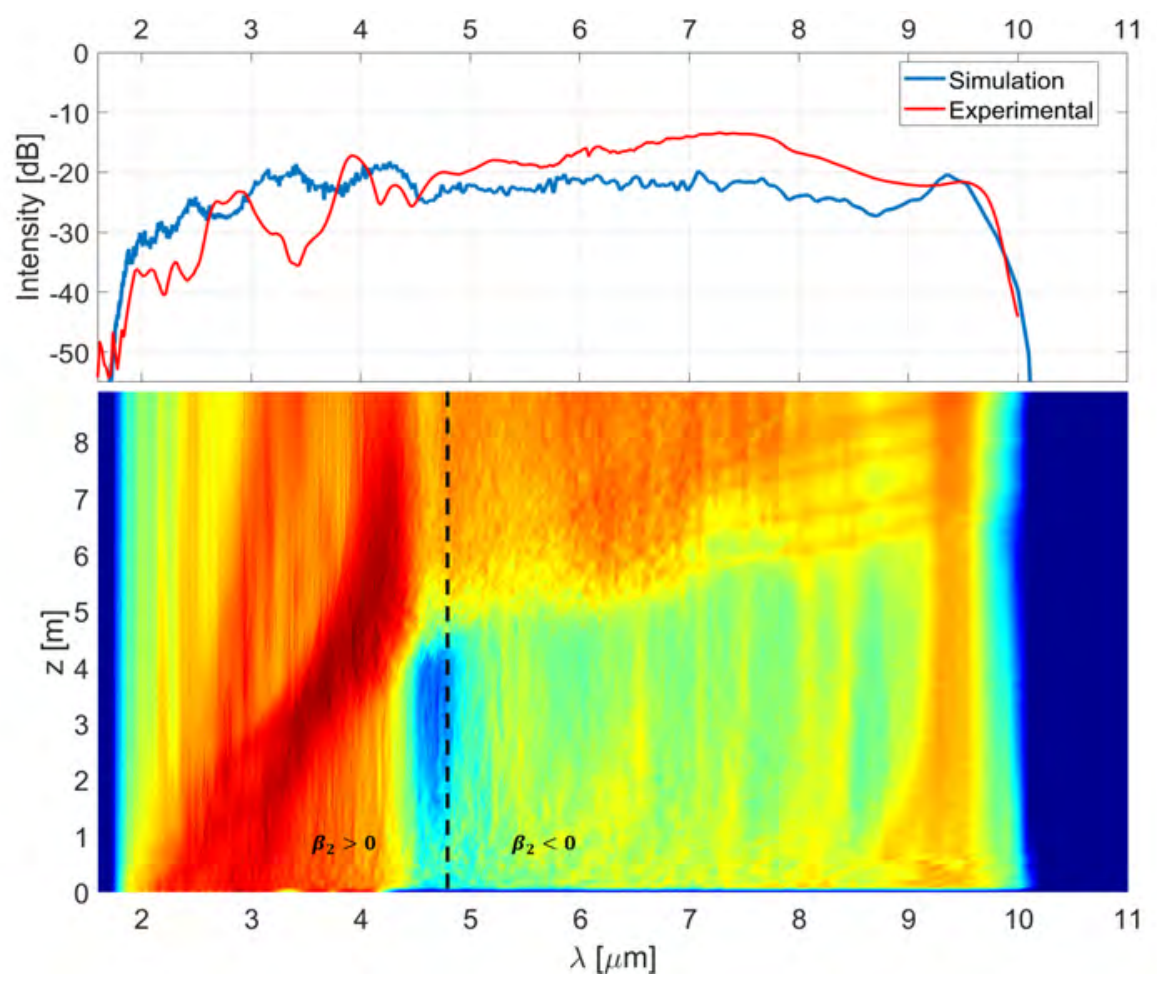

Figure 9: Full spectral evolution in the chalcogenide fiber with final output spectrum. 


\section{Conclusion and Recommendations}

In this work, we have developed a broadband fiber-based supercontinuum source spanning from 2 to $10 \mu \mathrm{m}$ with $16 \mathrm{~mW}$ output power. This was achieved using cascaded ZBLAN-chalcogenide optical fibers directly pumped by a compact 1550-nm pulsed fiber laser without using thulium-doped fiber amplifiers. We have further carried numerical simulations for broadband mid-infrared SC generation in a cascaded fiber system. Our simulations were consistent with the theory of quasi-CW SC generation. We show that noise amplification through MI and Raman gain leads to the break-up of the pulse envelope into a chaotic train of solitons which broadens the spectrum through Raman-induced self-frequency shift and dispersive waves. In terms of bandwidth, our numerical results are nearly identical to the experimentally measured spectra, both at the output of the ZBLAN fiber (within $0.05 \mu \mathrm{m}$ at $-20 \mathrm{~dB}$ level) and the chalcogenide fiber (within $0.04 \mu \mathrm{m}$ at $-20 \mathrm{~dB}$ level). The final output spectrum is a flat broadband spectrum covering the mid-IR from 2 to $10 \mu \mathrm{m}$. Further broadening is only limited by the transmission window of the chalcogenide fiber. Our main recommendation drawn from our numerical simulations would consist of using a shorter ZBLAN fiber (around $13 \mathrm{~m}$ ) and a chalcogenide microstructured fiber with a smaller core diameter (ideally $7 \mu \mathrm{m}$ ) [47]. This would shift its zero-dispersion wavelength towards shorter wavelengths $(<4 \mu \mathrm{m})$ and allow to fill the desired window (from 2 to $10 \mu \mathrm{m}$ ) with a few centimeters of fiber only (based on numerical simulations, See supplementary informations), while reducing the linear loss $(8.3 \mathrm{~dB})$. Our second recommendation consists into exploring different options for the infrared filtering system and to avoid free-space optics. Free-space optics and re-coupling to the chalcogenide fiber induces considerable losses in the system. An all-fiber filtering system, for example, would provide improved robustness and reduced losses. Antireflection coating or nanoimprinting of the chalcogenide fibers could also significantly reduce the Fresnel losses and the input damage threshold $[48,49]$. This would allow to considerably increase both the output power and the spectral power density. Another way to reduce free-space optics in this cascaded fiber setup is to use a fusion splice between the silica and the ZBLAN fiber, as demonstrated in Refs. [50,51]. Finally, we also recommend exploring telluride-glass fibers as a fourth fiber to add to the system. This would potentially allow to further broaden the spectrum and reach wavelengths beyond $10 \mu \mathrm{m}$ [52]. Polarization and coherence properties of the mid-IR cascaded SC light are further key parameters for some applications such as mid-IR OCT and interferometric measurements. We could for instance replace the fiber laser and the cascaded system with polarization-maintaining fibers [53,54]. About coherence, it is evident that tis cascaded fiber system does not maintain the initial temporal coherence of the input laser but we could consider all-normal dispersion (ANDi) chalcogenide fibers directly pumped with mid-IR femtosecond fiber lasers to improve this feature [44]. Nevertheless, we believe that, despite the mentioned weaknesses, current

mid-infrared supercontinuum all-fiber sources will find several applications and represent a landmark to foster the improvement of such broadband sources in other fiber or chip-based photonic platforms.

\section{$6 \quad$ Funding informations}

This project has received funding from and the European Union H2020 programme MSCA-ITN-SUPUVIR under grant agreement 722380 and the Agence Nationale de la Recherche (ANR) (I-SITE BFC ANR-15IDEX-0003, EIPHI Graduate School ANR-17-EURE-0002).

\section{Contributions and Disclosures}

All authors participate equally to the work. Disclosures: SV, LB, RC, CC (SelenOptics), FJ, SC, SP (LVF), GH (Leukos). All other authors (FS, AG, MM, JT, MR, JD, TS) declare no conflict of interest. 


\section{Supplementary informations}

In this section, we present simulation results for the same cascaded fiber system, but with a different chalcogenide fiber to study the effect of the zero-dispersion wavelength. The chosen fiber was presented by Saghaei et al. [47] in 2015 as part of an experimental study on mid-infrared SC generation. It is microstructured with the same air-hole pattern as the one used experimentally. Figure 10 shows a diagram of its cross-section. It is made of $\mathrm{As}_{2} \mathrm{Se}_{3}$ glass, which has similar nonlinear properties to $\mathrm{As}_{38} \mathrm{Se}_{62}$ glass. The main difference is its lower ZDW, which is at $3.81 \mu \mathrm{m}$. This is due to the different dimensions of its air-hole structure: it has a core diameter of $7 \mu \mathrm{m}$, a pitch of $5 \mu \mathrm{m}$, and an air-hole diameter of $3 \mu \mathrm{m}$.

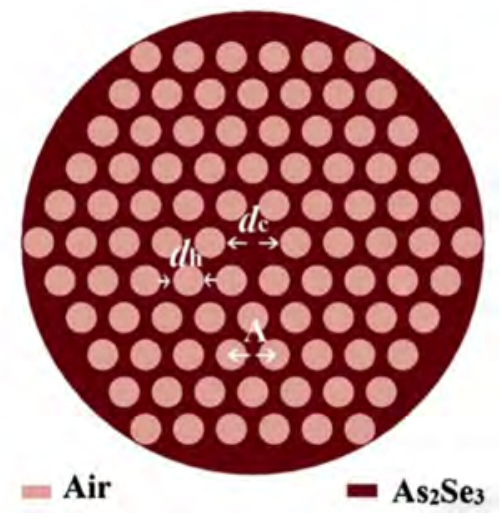

(a)

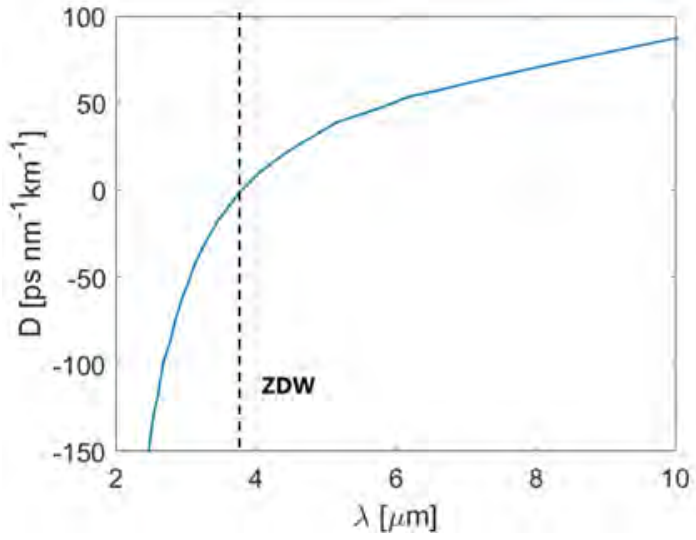

(b)

Figure 10: (a) Cross-section of the alternative chalcogenide fiber (adapted from [47]). (b) Dispersion curve.

Figure 11 shows the spectral evolution over $1 \mathrm{~cm}$ of this alternative chalcogenide fiber. This short propagation distance allowed to carry out the simulation with the filtered ZBLAN spectrum from a $460 \mathrm{ps}$ pump pulse. With the ZDW at $3.81 \mu \mathrm{m}$, a fraction of the filtered ZBLAN output spectrum is injected directly in the anomalous dispersion regime of this fiber (on the right of the dotted line in figure 11). This allows a great number of solitons to form directly without having to cross the ZDW barrier. Through SSFS, the solitons rapidly fill the transmission window with a relatively high-energy continuum. Therefore, by shifting the ZDW to $3.81 \mu \mathrm{m}$ with this alternative chalcogenide fiber, our simulations show that an almost flat SC spanning the $2-10 \mu \mathrm{m}$ window can be generated with only $1 \mathrm{~cm}$ of fiber.

The study of broadening dynamics in this fiber shows that, over the first centimeters of propagation, energy injected in the normal dispersion regime tends to stay concentrated on that side of the ZDW, and energy injected in the anomalous dispersion regime rapidly spreads to fill the transmission window. Therefore, we can postulate that shifting the ZDW further towards short wavelengths (with an even smaller core diameter) would help achieve an even flatter SC with only a few centimeters of fiber. This would reduce cost and the overall losses in the cascaded system. For practical applications however, a minimum length of $1 \mathrm{~m}$ is usually required at the source output. 

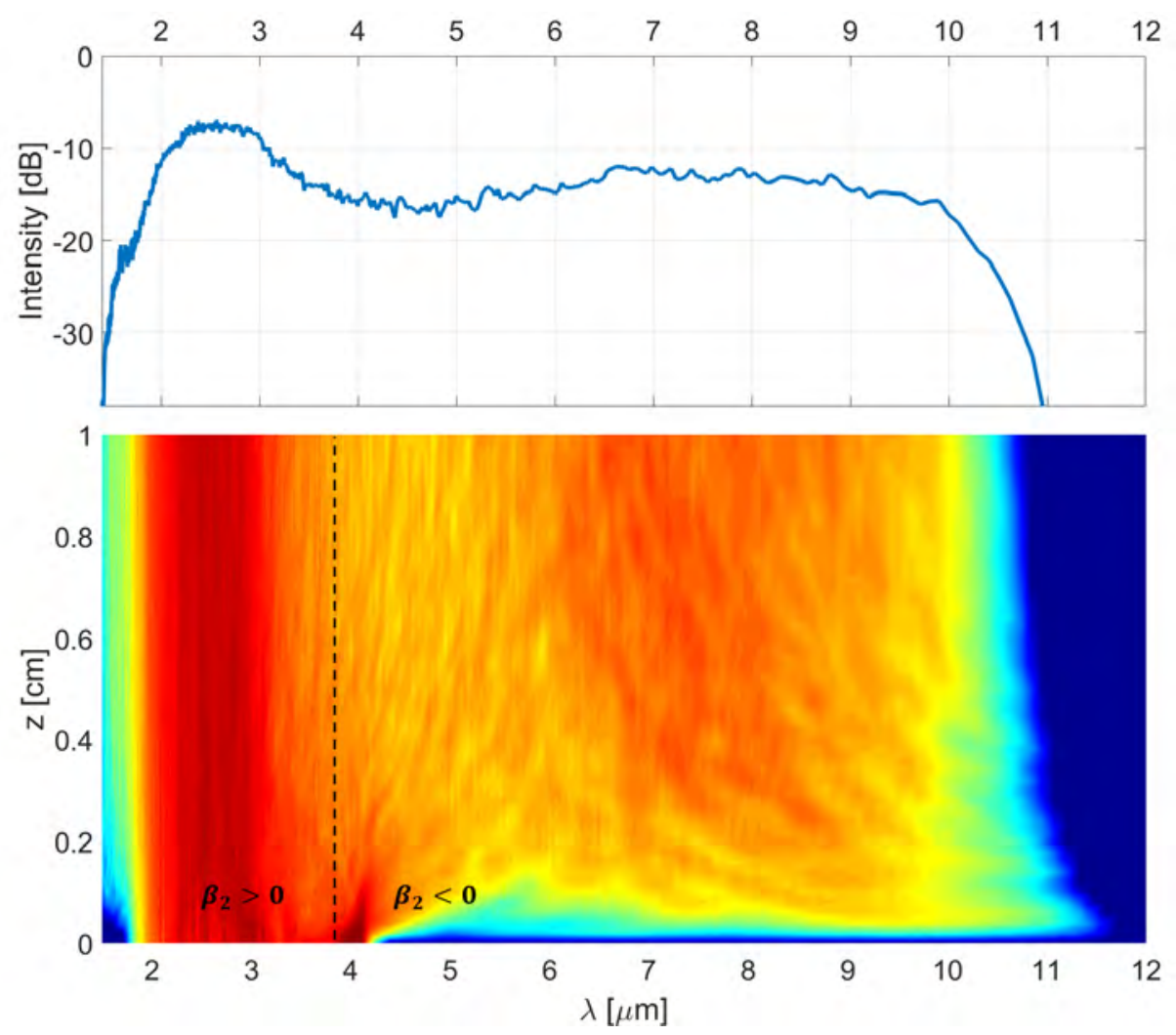

Figure 11: Spectral evolution in the $7 \mu \mathrm{m}$-core chalcogenide fiber. 


\section{References}

[1] R. R. Alfano, The Supercontinuum Laser Source: The Ultimate White Light (Springer, New York, 2016).

[2] J. M. Dudley and J. R. Taylor (Eds.) Supercontinuum generation in optical fibers. Cambridge University Press (2010).

[3] C. R. Petersen, U. Møller, I. Kubat, B. Zhou, S. Dupont, J. Ramsay, T. Benson, S. Sujecki, N. A. -Moneim, Z. Tang, D. Furniss, A. Seddon, and O. Bang, "Mid-infrared supercontinuum covering the 1.4-13.3 $\mu \mathrm{m}$ molecular fingerprint region using ultra-high NA chalcogenide step-index fibre", Nat. Photon. 8, 830-834 (2014).

[4] Yu, Y., Zhang, B., Gai, X., Zhai, C., Qi, S., Guo, W., Yang, Z., Wang, R., Choi, D., et al., "1.8-10 $\mu \mathrm{m}$ mid-infrared supercontinuum generated in a step-index chalcogenide fiber using low peak pump power," Optics Letters 40 (6), 1081-1084 (2015).

[5] T. Cheng, K. Nagasaka, T. H. Tuan, X. Xue, M. Matsumoto, H. Tezuka, T. Suzuki, and Y. Ohishi, "Mid-infrared supercontinuum generation spanning 2.0 to $15.1 \mu \mathrm{m}$ in a chalcogenide step-index fiber", Opt. Lett. 41 (9), 2117-2120 (2016).

[6] U.Møller, Y. Yu, I. Kubat, C. R. Petersen, X. Gai, L. Brilland, D. Mechin, C; Caillaud, J. Troles, B. Luther-Davies, and O. Bang, "Multi-milliwatt mid-infrared supercontinuum generation in a suspended core chalcogenide fiber," Opt. Express 23, 3282-3291 (2015).

[7] A. Lemière, F. Désévédavy, P. Mathey, P. Froidevaux, G. Gadret, J.-C. Jules, C. Aquilina, B. Kibler, P. Béjot, F. Billard, O. Faucher, and F. Smektala, "Mid-Infrared supercontinuum generation from 2 to $14 \mu \mathrm{m}$ in arsenic- and antimony-free chalcogenide glass fibers", J. Opt. Soc. Am. B 36, 183-192 (2019).

[8] C. R. Petersen, R. D. Engelsholm, C. Markos, L. Brilland, C. Caillaud, J. Troles, and O. bang, "Increased mid-infrared supercontinuum bandwidth and average power by tapering large-mode-area chalcogenide photonic crystal fibers", Opt. Express 25 (13), 15336-15347 (2017).

[9] Dai, Shixun and Wang, Yingying and Peng, Xuefeng and Zhang, Peiqing and Wang, Xunsi and Xu, Yinsheng, "A Review of Mid-Infrared Supercontinuum Generation in Chalcogenide Glass Fibers", Applied Sciences 8 (5), 707 (2018).

[10] A. N. Ghosh, M. Klimczak, R. Buczynski, J. M. Dudley, and T. Sylvestre, "Supercontinuum generation in heavy-metal oxide glass based suspended-core photonic crystal fibers", J. Opt. Soc. Am. B 35, 23112316 (2018).

[11] F. Belli, A. Abdolvand, W. Chang, J.C. Travers, and P. St. J. Russell, "Vacuum-ultraviolet to infrared supercontinuum in hydrogen-filled photonic crystal fiber", Optica 2 (4), 292-300 (2015).

[12] N. M. Israelsen, C. R. Petersen, A. Barh, D. Jain, M. Jensen, G. Hannesschläger, P. T. -Lichtenberg, C. Pedersen, A. Podoleanu, and Ole Bang, "Real-time high-resolution mid-infrared optical coherence tomography", Light: Science and Applications, 8, 11 (2019).

[13] I. Zorin, R. Su, A. Prylepa, J. Kilgus, M. Brandstetter, and B. Heise, "Mid-infrared Fourier-domain optical coherence tomography with a pyroelectric linear array", Opt. Express 26, 33428-33439 (2018). 
[14] J. Hult, R. S. Watt, and C. F. Kaminski, "High bandwidth absorption spectroscopy with a dispersed supercontinuum source", Opt. Express 15 (18), 11385-11395 (2007).

[15] C. Amiot, A. Aalto, P. Ryczkowski, J. Toivonen, and G. Genty, "Cavity enhanced absorption spectroscopy in the mid-infrared using a supercontinuum source", Appl. Phys. Lett. 111 (6), 061103 (2017).

[16] C. R. Petersen, P. M. Moselund, L. Huot, L. Hooper, and O. Bang, "Towards a table-top synchrotron based on supercontinuum generation", Infrared Physics and Technology 91, 182-186 (2018).

[17] A. B. Seddon, "Biomedical Applications in Probing Deep Tissue Using Mid-Infrared Supercontinuum Optical Biopsy" in book "Deep Imaging in tissue and biomedical Media" by L. Shi and R. R. Alfano (Pan Stanford Publishing, Singapore, pp 231-294 (2016).

[18] C. R. Petersen, N. Prtljaga, M. Farries, J. Ward, B. Napier, G. R. Lloyd, J. Nallala, N. Stone, and O. Bang, "Mid-infrared multispectral tissue imaging using a chalcogenide fiber supercontinuum source", Opt. Lett. 43(5), 999-1002 (2018).

[19] F. Désévédavy, G. Gadret, J. -C. Jules, B. Kibler, and F. Smektala, "Supercontinuum Generation in Tellurite Optical Fibers", Technological Advances in Tellurite Glasses, Springer Ser. Mater. Sci., vol 254, Springer (2017).

[20] P. Lucas, Z. Yang, M. K. Fah, T. Luo, S. Jiang, C. Boussard-Pledel, M.-L. Anne, and B. Bureau, "Telluride glasses for far infrared photonic applications," Opt. Mater. Express 3, 1049-1058 (2013).

[21] Z. Zhao, B. Wu, X. Wang, Z. Pan, Z. Liu, P. Zhang, X. Shen, Q. Nie, S. Dai, and R. Wang, "Midinfrared supercontinuum covering $2.0-16 \mu \mathrm{m}$ in a low-loss telluride single-mode fiber", Laser Photonics Rev. 11, No. 2 (2017).

[22] C. Xia, M. Kumar, O. P. Kulkarni, M. N. Islam, F. L. Terry, M. J. Freeman, M. Poulain, and G. Mazé, "Mid-infrared supercontinuum generation to $4.5 \mu \mathrm{m}$ in ZBLAN fluoride fibers by nanosecond diode pumping", Opt. Lett. 31 (17), 2553-2555 (2006).

[23] M. Michalska, P. Hlubina, and J. Swiderski, "Mid-infrared Supercontinuum Generation to $4.7 \mu \mathrm{m}$ in a ZBLAN Fiber Pumped by an Optical Parametric Generator", IEEE Photon. J. 9 (2), 3200207 (2017).

[24] C. Agger, C. Petersen, S. Dupont, H. Steffensen, J. K. Lyngsø, C. L. Thomsen, J. Thøgersen, S. R. Keiding, and O. Bang, "Supercontinuum generation in ZBLAN fibers - detailed comparison between measurement and simulation", J. Opt. Soc. Am. B 29 (4), 635-645 (2012).

[25] C. L. Hagen, J.W. Walewski, and S.T. Sanders, "Generation of a continuum extending to the midinfrared by pumping ZBLAN fiber with an ultrafast $1550 \mathrm{~nm}$ source", IEEE Phot. Tech. Lett. 18, 91-93 (2006).

[26] C. Xia, M. Kumar, M.-Y. Cheng, R. S. Hegde, M. N. Islam, A. Galvanauskas, H. G. Winful, F. L. Terry, M. J. Freeman, M. Poulain, and G. Mazé, "Power scalable mid-infrared supercontinuum generation in ZBLAN fluoride fibers with up to 1.3 watts time-averaged power," Opt. Express 15, 865-871 (2007).

[27] Darren D. Hudson, Sergei Antipov, Lizhu Li, Imtiaz Alamgir, Tomonori Hu, Mohammed El Amraoui, Younes Messaddeq, Martin Rochette, Stuart D. Jackson, and Alexander Fuerbach, "Toward all-fiber supercontinuum spanning the mid-infrared," Optica 4, 1163-1166 (2017). 
[28] F. Theberge, N. Berube, S. Poulain, S. Cozic, S. Chatigny, L.-R. Robichaud, L.-P. Pleau, M. Bernier, and R. Vallee, "Infrared supercontinuum generated in concatenated InF3 and As2Se3 fibers", Opt. Express 26 (11), 13952-13960 (2018).

[29] R. A. Martinez, G. Plant, K. Guo, B. Janiszewski, M. J. Freeman, R. L. Maynard, M. N. Islam, F. L. Terry, O. Alvarez, F. Chenard, R. Bedford, R. Gibson, and A. I. Ifarraguerri, "Mid-infrared supercontinuum generation from 1.6 to $>11 \mu \mathrm{m}$ using concatenated step-index fluoride and chalcogenide fibers", Opt. Lett. 43 (2), 296-299 (2018).

[30] R. A. Martinez, K. Guo, T. Zhai, F. L. Terry, L. E. Pierce, M. N. Islam, R. Gibson, J. M. Reed, R. G. Bedford, L. Maksymiuk, M. J. Freeman, B. A. Gorin, N. P. Christian, and A. I. Ifarraguerri, "Active Mid-Wave to Long-Wave Supercontinuum FTIR Sensor for Standoff Chemical Detection," J. Lightwave Technol. 37, 3626-3636 (2019).

[31] I. Kubat, C. R. Petersen, U. Visbech Møller, A. Seddon, T. Benson, L. Brilland, D. Méchin, P. M. Moselund, and O. Bang, "Thulium pumped mid-infrared 0.9-9 $\mu \mathrm{m}$ supercontinuum generation in concatenated fluoride and chalcogenide glass fibers," Opt. Express 22, 3959-3967 (2014).

[32] C. R. Petersen, P. M. Moselund, C. Petersen, U. Møller, and O. Bang, "Spectral-temporal composition matters when cascading supercontinua into the mid-infrared," Opt. Express 24, 749-758 (2016).

[33] C. R. Petersen, P. M. Moselund, Ch. Petersen, U. Møller, O. Bang, "Mid-IR supercontinuum generation beyond $7 \mu \mathrm{m}$ using a silica-fluoride-chalcogenide fiber cascade," Proc. SPIE 9703, Optical Biopsy XIV: Toward Real-Time Spectroscopic Imaging and Diagnosis, 97030A (2016).

[34] L.-R. Robichaud, V. Fortin, J.-C. Gauthier, S. Châtigny, J.-F. Couillard, J.-L. Delarosbil, R. Vallée, and M. Bernier, "Compact 3-8 $\mu \mathrm{m}$ supercontinuum generation in a low-loss As2Se3 step-index fiber," Opt. Lett. 41, 4605-4608 (2016).

[35] Jinmei Yao, Bin Zhang Ke Yin, and Jing HouJinmei, "The 2- $\mu \mathrm{m}$ to 6- $\mu \mathrm{m}$ mid-infrared supercontinuum generation in cascaded ZBLAN and As2Se3 step-index fibers," Chinese Phys. B 28084209 (2019).

[36] http://norblis.com/

[37] J. M. Dudley, G. Genty, S. Coen, "Supercontinuum generation in photonic crystal fiber," Reviews of Modern Physics 78(4), 1135 (2006).

[38] G. P. Agrawal, Nonlinear fiber optics, fourth edition, Academic Press, Elsevier, San Diego (2007).

[39] O. V. Sinkin, R. Holzlohner, J. Zweck, C.R. Menyuk, "Optimization of the split-step Fourier method in modeling optical-fiber communications systems," Journal of lightwave technology 21(1), 61-68 (2003).

[40] B. Kibler, J. M. Dudley, S. Coen, "Supercontinuum generation and nonlinear pulse propagation in photonic crystal fiber: influence of the frequency-dependent effective mode area," Applied Physics B, 81(2-3), 337-342 (2005).

[41] ZFG Singlemode. (n.d.). https://leverrefluore.com/products/passive-fibers/zfg-singlemode/

[42] Fluoride fiber technology. (n.d.). Retrieved from https://www.fiberlabs.com/glossary/technology/

[43] A. D. Sánchez, P. I. Fierens, S. M. Hernandez, J. Bonetti, G. Brambilla, D. F. Grosz, "Anti-Stokes Raman gain enabled by modulation instability in mid-IR waveguides. J. Opt. Soc. Am. B, 35(11), 2828-2832 (2018). 
[44] E. Genier, P. Bowen, T. Sylvestre, J. M. Dudley, P. Moselund, O. Bang, "Amplitude noise and coherence degradation of femtosecond supercontinuum generation in all-normal-dispersion fibers," J. Opt. Soc. Am. B, 36(2), 161-167 (2019).

[45] Q. Coulombier, L. Brilland, P. Houizot, T. Chartier, T. N. NGuyen, F. Smektala, G. Renversez, A. Monteville, D. Mechin, T. Pain, H. Orain, J.-C. Sangleboeuf, and J. Troles, "Casting method for producing low-loss chalcogenide microstructured optical fibers," Opt. Express 18, 9107-9112 (2010).

[46] G. Lenz, J. Zimmermann, T. Katsufuji, M. E. Lines, H. Y. Hwang, S. Spälter, R. E. Slusher, S.-W. Cheong, J. S. Sanghera, and I. D. Aggarwal, "Large Kerr effect in bulk Se-based chalcogenide glasses," Opt. Lett. 25, 254-256 (2000).

[47] H. Saghaei, M. Ebnali-Heidari, M. K. Moravvej-Farshi, "Mid-infrared supercontinuum generation via $\mathrm{As}_{2} \mathrm{Se}_{3}$ chalcogenide photonic crystal fibers," Applied Optics 54(8), 2072-2079 (2015).

[48] L.-R. Robichaud, S. Duval, L.-P. Pleau, V. Fortin, S. Toubou Bah, S. Châtigny, R. Vallée, and M. Bernier, "High-power supercontinuum generation in the mid-infrared pumped by a soliton selffrequency shifted source," Opt. Express 28, 107-115 (2020).

[49] C. R. Petersen, M. B. Lotz, G. Woyessa, A. N. Ghosh, T. Sylvestre, L. Brilland, J. Troles, M. H. Jakobsen, R. Taboryski, and O. Bang, "Nanoimprinting and tapering of chalcogenide photonic crystal fibers for cascaded supercontinuum generation," Opt. Lett. 44, 5505-5508 (2019).

[50] K. Yin, B. Zhang, J. Yao, L. Yang, S. Chen, and J. Hou "Highly stable, monolithic, single-mode mid-infrared supercontinuum source based on low-loss fusion spliced silica and fluoride fibers," Opt. Lett. 41, 946-949 (2016).

[51] Z. Zheng, D. Ouyang, J. Zhao, M. Liu, S. Ruan, P. Yan, and J. Wang "Scaling all-fiber mid-infrared supercontinuum up to $10 \mathrm{~W}$-level based on thermal-spliced silica fiber and ZBLAN fiber," Photon. Res. 4, 135-139 (2016).

[52] S. Cui, C. Boussard-Plédel, J. Troles, B. Bureau, "Telluride glass single mode fiber for mid and far infrared filtering," Optical Materials Express, 6(4), 971-978 (2016).

[53] S. A. Rezvani, Y. Nomura, K. Ogawa, and T. Fuji, "Generation and characterization of mid-infrared supercontinuum in polarization maintained ZBLAN fibers," Opt. Express 27, 24499-24511 (2019).

[54] A. N. Ghosh, M. Meneghetti, C. R. Petersen, O. Bang, L. Brilland, S. Venck, J. Troles, J. M. Dudley, and T Sylvestre, "Chalcogenide-glass polarization-maintaining photonic crystal fiber for mid-infrared supercontinuum generation," Journal of Physics: Photonics, 1(4), 044003 (2019). 